\title{
Erratum to: The Universal C*-Algebra of the Electromagnetic Field
}

DETLEV BUCHHOLZ ${ }^{1}$, FABIO CIOLLI ${ }^{2}$, GIUSEPPE RUZZI $^{2}$ and EZIO VASSELLI ${ }^{2}$

${ }^{1}$ Institut für Theoretische Physik, Universität Göttingen, Friedrich-Hund-Platz 1, 37077 Göttingen, Germany.e-mail: buchholz@theorie.physik.uni-goettingen.de ${ }^{2}$ Dipartimento di Matematica, Universitá di Roma "Tor Vergata", Via della Ricerca Scientifica 1, 00133 Rome, Italy

Published online: 22 December 2015 - (C) Springer Science+Business Media Dordrecht 2015

\section{Erratum to: Lett Math Phys \\ DOI 10.1007/s11005-015-0801-y}

The original version of this article unfortunately contained a mistake. The presentation of Eq. (2.3) was incorrect. The corrected equation is given below.

$$
\left\lfloor V(a, g),\left\lfloor V\left(a_{1}, g_{1}\right), V\left(a_{2}, g_{2}\right)\right\rfloor=1 \quad \text { if } \quad \operatorname{supp} g_{1} \perp \operatorname{supp} g_{2}\right.
$$

